\title{
Frankrijk
}

\section{Länderbericht Frankreich}

\section{B. Crocq*}

Nach einer kurzen Einleitung (1) werden wir uns mit den Themen der Stellung des überlebenden Ehegatten (2) und des Pflichtteilsrechts (3) beschäftigen.

\section{Einleitung}

Zur Einleitung bietet sich zunächst ein kurzer Überblick über die historische Entwicklung, die Rechtsquellen (1.1) sowie über das internationale Erbrecht (1.2) an.

\subsection{Historische Entwicklung und Rechtsquellen}

Das Erbrecht ist im 3. Buch des französischen Zivilgesetzbuchs - Code civil - geregelt (Art. 720 ff.). Seit dem Inkrafttreten des Code civil im Jahre 1804 wurde das Erbrecht in seinen Grundsätzen wenig geändert.

Zwei wichtige Reformen sind hier zu erwähnen:

- Das Gesetz des 3. Dezember 2001, in Kraft getreten am 1. Juli $2002^{1}$ sowie

- Das Gesetz des 23. Juni 2006, in Kraft getreten am 1. Januar $2007 .^{2}$

Das Gesetz des 3. Dezember 2001 hat die Stellung des überlebenden Ehegatten wesentlich gestärkt. Vor dem 1. Juli 2002 war die Stellung des überlebenden Ehegatten relativ schwach (in den meisten Fällen hatte dieser nur ein Nießbrauchrecht). Dies wurde aber damit ausgeglichen, dass der überlebende Ehegatte kraft des gesetzlichen Güterstandes (Errungenschaftsgemein-

Barbara Crocq ist Juristin im Bereich internationales Privatrecht und Rechtsvergleichung, CRIDON Lyon

1. Loi $n^{\circ}$ 2001-1135 du 3 décembre 2001 relative aux droits du conjoint survivant et des enfants adultérins et modernisant diverses dispositions de droit successoral.

2. Loi $n^{\circ} 2006-728$ du 23 juin 2006 portant réforme des successions et des libéralités. schaft) umfassend am Vermögen des Erblassers beteiligt war.

Das Gesetz des 23. Juni 2006 hat weitere Änderungen mit sich gebracht und insbesondere das Pflichtteilsrecht wesentlich beeinträchtigt.

Außerdem hat das "Modernisierungsgesetz" vom 18. November $2016^{3}$ (in Kraft getreten am 1. November 2017) bestimmte Aspekte des Erbrechts neugestaltet: die Besitzeinweisung (envoi en possession) des Universalvermächtnisnehmers, die Nachlassoption (option successorale) und die Erblosigkeit (successions vacantes). ${ }^{4}$ Diese Aspekte werden allerdings im Rahmen dieses Berichts nicht behandelt.

\subsection{Kollisionsnormen}

Vor dem Inkrafttreten der europäischen Erbrechtsverordnung (EuErbVO $)^{5}$ galt im französischen internationalen Privatrecht eine Nachlassspaltung:

- Unbewegliches Vermögen war an des Recht des Belegenheitsortes (lex rei sitae) angeknüpft;

- Bewegliches Vermögen war an das Recht des letzten Wohnsitzes des Erblassers angeknüpft.

Seit dem 17. August 2015 wird das anwendbare Recht nach Artikel 21 und 22 EuErbVO bestimmt. Es gilt nun im Prinzip eine Nachlasseinheit.

Wenn das französische Recht gemäß der EuErbVO zur Anwendung kommt, sind die nachstehenden Ausführungen dann beispielsweise auch für niederländische Notare relevant.

3. Loi $n^{\circ}$ 2016-1547 du 18 novembre 2016 de modernisation de la justice du XXle siècle.

4. Bruno Jeannet, "La retouche du droit des successions par la loi de modernisation de la justice du XXlème siècle ", Cahier du CRIDON Lyon $n^{\circ} 80$, février 2018.

5. Verordnung (EU) Nr. 650/2012 des Europäischen Parlaments und des Rates vom 4. Juli 2012 über die Zuständigkeit, das anzuwendende Recht, die Anerkennung und Vollstreckung von Entscheidungen und die Annahme und Vollstreckung öffentlicher Urkunden in Erbsachen sowie zur Einführung eines Europäischen Nachlasszeugnisses. 


\section{Stellung des überlebenden Ehegatten}

Vor der erbrechtlichen Auseinandersetzung muss der Güterstand abgewickelt werden (2.1). Zu unterscheiden ist dann zwischen der gesetzlichen Erbfolge (2.2) und der gewillkürten Erbfolge (2.3).

\subsection{Güterrechtliche Auseinandersetzung}

Mit der güterrechtlichen Auseinandersetzung wird bestimmt, was der überlebende Ehegatte kraft des Güterstandes erlangt und was zum Nachlass gehört. Der Güterstand endet mit dem Tod eines Ehegatten (Art. 1441 des Code civil). Es gibt keine fortgesetzte Gütergemeinschaft im französischen Recht (Art. 1442 des Code civil).

Der französische gesetzliche Güterstand ist die Errungenschaftsgemeinschaft (communauté de biens réduite aux acquêts) gemäß Art. 1400 ff. des Code civil. Zu unterscheiden sind die Sondergüter (biens propres) und die Gesamtgüter (biens communs). Als Gesamtgüter sind in der Regel die Güter zu betrachten, die ein oder beide Ehegatten während der Ehe entgeltlich erworben haben. Wenn der gesetzliche Güterstand abgewickelt wird, erhält jeder Ehegatte seine Sondergüter zurück. Das Gesamtgut wird in zwei gleichwertige Teile als Bruchteilsgemeinschaft geteilt: Der überlebende Ehegatte erhält eine Hälfte, die andere Hälfte fällt in den Nachlass.

Den Ehegatten steht die Möglichkeit zu, diese Auseinandersetzungsvorschriften durch einen Ehevertrag abzuändern. In der französischen Notarpraxis sind güterrechtliche Vorteilszuweisungen unter Ehegatten (avantages matrimoniaux) häufig. Zum Beispiel können die Ehegatten in ihrem Ehevertrag vereinbaren, dass das ganze Gesamtgut dem überlebenden Ehegatten zufallen soll (clause d'attribution intégrale de la communauté au conjoint survivant).

Solche Vereinbarungen, die als avantages matrimoniaux bezeichnet werden, werden nicht als Schenkung behandelt (Art. 1527 des Code civil). Den gemeinsamen Kindern beider Ehegatten steht in diesem Fall keine Herabsetzungsklage wegen Verletzung des Pflichtteils zu. Lediglich der Pflichtteil der nicht gemeinsamen Kinder wird gemäß Art. 1527 des Code civil geschützt (action en retranchement).

Was nach der güterrechtlichen Auseinandersetzung in den Nachlass fallt, unterliegt dann den erbrechtlichen Vorschriften des Code civil.

\subsection{Gesetzliche Erbfolge}

Unter bestimmten Voraussetzungen (2.2.1) stehen dem Ehegatten ein gesetzliches Erbteil (2.2.2) sowie weitere Rechte (2.2.3) zu.

\subsubsection{Voraussetzung des gesetzlichen Erbrechts des} Ehegatten

Voraussetzung des gesetzlichen Erbrechts des überlebenden Ehegatten ist das Bestehen einer gültigen Ehe mit dem Erblasser bis zu dessen Tod (Art. 732 des Code civil). Dies gilt auch für gleichgeschlechtliche Ehen, die seit dem Gesetz des 17. Mai 2013 im französischen Recht erlaubt sind. ${ }^{6}$

Nur die rechtskräftige Scheidung schließt das Erbrecht des überlebenden Ehegatten aus. Das gesetzliche Erbrecht bleibt bei einem rechtshängigen Scheidungsverfahren sowie bei einem rechtskräftigen Urteil auf Trennung von Tisch und Bett (séparation de corps) bestehen.

Im Gegensatz zum Ehegatten hat der eingetragene Lebenspartner (partenaire pacsé) kein gesetzliches Erbrecht. ${ }^{7}$ Es steht ihm lediglich ein einjähriges Wohnungsrecht zu (Art. 515-6 Abs. 3 des Code civil). Er kann aber durch ein Testament begünstigt werden und ist dann auf steuerrechtlicher Ebene dem Ehegatten umfassend gleichgestellt.

\subsubsection{Umfang des Ehegattenerbrechts}

Der überlebende Ehegatte wird entweder allein oder neben Blutverwandten (héritiers par le sang) am Nachlass beteiligt (Art. 756 des Code civil).

Hinterlässt der Erblasser Abkömmlinge (Erben der 1. Ordnung gemäß Art. 734 des Code civil), so wird unterschieden, ob der überlebende Ehegatte ausschließlich mit gemeinsamen Kindern beider Ehepartner oder auch mit einseitigen Kindern des verstorbenen Ehegatten konkurriert (Art. 757 des Code civil).

Sind ausschließlich gemeinsame Kinder vorhanden, hat der überlebende Ehegatte ein Wahlrecht: Er erhält entweder den Nießbrauch des gesamten Nachlasses oder das Eigentum an einem Viertel (1/4) des Nachlasses. Übt der überlebende Ehegatte sein Wahlrecht nicht innerhalb von drei Monaten nach schriftlicher Aufforderung durch einen Miterben aus, so gilt dies als konkludente Entscheidung für den Nießbrauch (Art. 758-3 des Code civil). Verstirbt er, ohne sein Wahlrecht ausgeübt zu haben, gilt ebenfalls der Nießbrauch als angenommen (Art. 758-4 des Code civil). Mit dem Tod des überlebenden Ehegatten erlischt der Nießbrauch, sodass der Nachlass anschließend zwischen den Abkömmlingen aufgeteilt wird. Der Nießbrauch kann in eine Leibrente (Art. 759 des Code civil) oder in einen Geldbetrag (Art. 761 des Code civil) umgewandelt werden.

Hinterlässt der Erblasser mindestens ein einseitiges Kind, entfällt das Wahlrecht und der überlebende Ehegatte hat lediglich Anspruch auf das Eigentum an einem Viertel (1/4) des Nachlasses. Das vorgenannte Wahlrecht kann dem überlebenden Ehegatten jedoch durch letztwillige Verfügung wieder eingeräumt werden.

Hinterlässt der Erblasser keine Abkömmlinge, aber beide Elternteile, erhält der überlebende Ehegatte das Eigentum an der Hälfte des Nachlasses (Art. 757-1 des Code civil). Die andere Hälfte wird zwischen den Eltern des Erblassers aufgeteilt. Ist ein Elternteil weggefallen, geht dessen Erbteil von einem Viertel (1/4) dem überlebenden Ehegatten zu.

6. Loi $n^{\circ}$ 2013-404 du 17 mai 2013 ouvrant le mariage aux couples de personnes de même sexe.

7. In Frankreich ist die eingetragene Lebenspartnerschaft (pacte civil de solidarité - PACS) durch Art. 515-1 ff. des Code civil geregelt. 
Hinterlässt der Erblasser weder Abkömmlinge noch seine Eltern, erhält der überlebende Ehegatte den gesamten Nachlass (Art. 757-2 des Code civil).

Somit werden alle Seitenverwandten durch den überlebenden Ehegatten von der Erbfolge ausgeschlossen. Es besteht jedoch ein gesetzliches Rückfallrecht (droit de retour) zugunsten der Geschwister. Sind die Eltern des Erblassers vorverstorben und hinterlässt dieser keine Abkömmlinge, haben seine Geschwister (beziehungsweise deren Abkömmlinge) Anspruch auf die Hälfte der Gegenstände, die der Erblasser durch Schenkung von Todes wegen von seinen Aszendenten erhalten hat und die sich noch in natura im Nachlass befinden, sofern die Geschwister von den Aszendenten abstammen, von denen der Erblasser die Gegenstände erhalten hat (Art. 757-3 des Code civil).

\subsubsection{Weitere Rechte des überlebenden Ehegatten}

Dem überlebenden Ehegatten stehen Nutzungsrechte an der Ehewohnung (2.2.3.1) sowie ein Unterhaltsanspruch (2.2.3.2) zu.

\subsubsection{Nutzungsrechte an der Ehewohnung} $\mathrm{Zu}$ unterscheiden ist das einjährige Nutzungsrecht (2.2.3.1.1) und das Dauernutzungsrecht (2.2.3.1.2).

\subsection{Einjähriges Nutzungsrecht}

Gemäß Art. 763 des Code civil hat der überlebende Ehegatte für die Dauer eines Jahres ein unentgeltliches Nutzungsrecht an der Ehewohnung und dem Mobiliar. Der überlebende Ehegatte muss die Ehewohnung zum Zeitpunkt des Erbfalls tatsächlich als Hauptwohnung genutzt haben und die Wohnung muss Eigentum beider Ehegatten gewesen sein oder zum Nachlass gehören. Handelt es sich bei der Ehewohnung um eine Mietwohnung, so hat der überlebende Ehegatte für die Dauer eines Jahres Anspruch auf Zahlung des Mietzinses aus dem Nachlass.

Das einjährige Nutzungsrecht an der Ehewohnung wird als direkte Ehewirkung und nicht als Erbrecht bezeichnet. Es zählt zum ordre public, das heißt, es kann weder durch Testament entzogen noch durch Vereinbarung der Ehegatten ausgeschlossen werden. Der überlebende Ehegatte kann lediglich nach dem Erbfall darauf verzichten.

\subsection{Dauernutzungsrecht}

Gemäß Art. 764 des Code civil hat der überlebende Ehegatte ein lebenslanges Wohn- und Nutzungsrecht an Ehewohnung und Mobiliar, falls dieses zum Nachlass gehört.

Im Unterschied zum einjährigen Wohnrecht hat der Erblasser die Möglichkeit, dem überlebenden Ehegatten das lebenslange Wohn- und Nutzungsrecht an Ehewohnung und Mobiliar zu entziehen, da dieses nicht zum ordre public gehört. Die Entziehung kann allerdings nur durch ein öffentliches Testament gemäß Art. 971 des Code civil erfolgen. Eine allgemeine Enterbung des überlebenden Ehegatten reicht nicht aus, sie muss sich ausdrücklich auf das lebenslange Wohn- und Nutzungsrecht beziehen.
Das Dauernutzungsrecht wird als Erbrecht betrachtet. Daher muss der überlebende Ehegatte tatsächlich Erbe werden, um es beanspruchen zu können. Der überlebende Ehegatte muss innerhalb eines Jahres ab dem Erbfall seinen Willen, von dem lebenslangen Wohnund Nutzungsrecht Gebrauch zu machen, zum Ausdruck bringen (Art. 765-1 des Code civil).

Der Wert des Wohn- und Nutzungsrechts ist auf den Erbteil des überlebenden Ehegatten anzurechnen (Art. 765 des Code civil). Ist der Wert des Wohn- und Nutzungsrechts geringer als der Wert des Erbteils des überlebenden Ehegatten, hat er Anspruch auf eine Nachlassbeteiligung in Höhe des Differenzbetrags. Ist der Wert des Wohn- und Nutzungsrechts höher als der seines Erbteils, ist der überlebende Ehegatte nicht verpflichtet, den Differenzbetrag auszugleichen und an den Nachlass zu zahlen.

\subsubsection{Unterhaltsanspruch}

Gemäß Art. 767 des Code civil steht einem bedürftigen Ehegatten ein Unterhaltsanspruch gegen den Nachlass zu. Er muss diesen Anspruch innerhalb eines Jahres ab dem Erbfall oder ab dem Zeitpunkt, zu dem die Erben keine Zahlungen mehr an ihn leisten, geltend machen, wobei sich diese Frist bis zur Teilung verlängern kann. Der Unterhaltsanspruch richtet sich gegen den Nachlass. Falls der vorhandene Nachlass nicht ausreicht, um den Unterhaltsanspruch zu erfüllen, können neben den Erben auch Vermächtnisnehmer zur Erfüllung des Anspruchs herangezogen werden.

Der überlebende Ehegatte ist also vom Gesetz geschützt. Seine Rechte können zudem im Rahmen einer gewillkürten Erbfolge verbessert werden.

\subsection{Gewillkürte Erbfolge}

Der überlebende Ehegatte kann entweder durch ein Testament (2.3.1) oder durch eine vertragliche Erbeinsetzung (2.3.2) bevorzugt werden.

\subsubsection{Testamente}

Im Hinblick auf Testamente ist es sinnvoll, sowohl die Formvoraussetzungen (2.3.1.1) als auch den Inhalt (2.3.1.2) der Testamente zu betrachten.

\subsubsection{Art und Form}

Das französische Recht kennt drei Formen ordentlicher Testamente (Art. 969 ff. des Code civil):

- Das eigenhändige Testament (testament olographe): Dieses muss vom Erblasser eigenhändig niedergeschrieben, datiert und unterzeichnet sein. Es kann auch in einer fremden Sprache verfasst sein.

- Das öffentliche Testament muss vom Erblasser dem Notar im Beisein entweder eines zweiten Notars oder von zwei Zeugen diktiert worden sein. Seit einer Reform des 16. Februar 2015 ist die Möglichkeit der Mitwirkung eines Dolmetschers ausdrücklich vorgesehen. ${ }^{8}$

8. Loi $n^{\circ}$ 2015-177 du 16 février 2015 relative à la modernisation et à la simplification du droit et des procédures dans les domaines de la justice et des affaires intérieures. 
- Das verschlossene oder "mystische" Testament (in der Praxis selten gebraucht).

Öffentliche Testamente sowie bei einem Notar hinterlegte Privattestamente werden im zentral geführten Testamentsregister (fichier central des dispositions de dernières volontés) erfasst.

Jedes Testament ist frei widerruflich, entweder durch eine ausdrückliche Willenserklärung oder konkludent über die Errichtung eines Testamentes, dessen Bestimmungen mit dem ursprünglichen Testament unvereinbar sind.

Gemeinschaftliche Testamente sind gemäß Art. 968 des Code civil verboten.

\subsubsection{Inhalt der Testamente}

Der Erblasser kann in Form von Vermächtnissen über sein Vermögen verfügen. Es gibt drei Arten von Vermächtnissen:

- Das Universalvermächtnis (legs universel): Nach Art. 1003 des Code civil handelt es sich um die Zuwendung des gesamten Vermögens, das beim Tod des Erblassers vorhanden ist, an eine oder mehrere Personen. Allein die eventuelle Berufung auf die Gesamtheit des Vermögens ist ausschlaggebend. Ob der Universalvermächtnisnehmer tatsächlich den gesamten Nachlass erhält, ist irrelevant.

- Das Erbteilvermächtnis (legs à titre universel): Nach Art. 1010 des Code civil liegt ein Erbteilvermächtnis dann vor, wenn der Erblasser dem Vermächtnisnehmer einen Bruchteil des frei verfügbaren Vermögens, alle Immobilien oder alle Mobilien oder einen Teil davon zuwendet. Diese Aufzählung ist limitativ.

- Das Einzel- oder Stückvermächtnis (legs particulier ou à titre particulier): Die Definition desselben ergibt sich implizit aus Art. 1010 Abs. 2. Es handelt sich um jedes Vermächtnis, das weder ein Universalvermächtnis noch ein Erbteilvermächtnis darstellt.

Vermächtnisse können an bestimmte Bedingungen oder Auflagen geknüpft sein.

Substitutionen sind im französischen Recht grundsätzlich verboten (prohibition des substitutions fidéicommissaires); sie sind nur in bestimmten Fällen vom Gesetz ausdrücklich gestattet. Seit der Reform des 23. Juni 2006 sind folgende Substitutionen erlaubt:

- Die nicht-befreite Vor- und Nacherbschaft (libéralité graduelle): Nach Art. 1048 des Code civil handelt es sich um eine Zuwendung (testamentarische Verfügung oder Schenkung) an einen Begünstigten bzw. einen Vermächtnisnehmer mit der Auflage, das ihm zugewandte Vermögen zu erhalten, und mit der Maßgabe, bei seinem Tod einen im Testament oder Vertrag genannten Nacherben zu begünstigen. Falls die Nacherbschaft eintritt, wird der Nacherbe behandelt, als habe er seine Rechte unmittelbar vom Verfügenden erhalten.

- Die befreite Vor- und Nacherbschaft (libéralité résiduelle): Nach Art. 1057 des Code civil handelt es sich um die Schenkung oder das Vermächtnis an einen Vorerben mit der Auflage, die beim Tode des Begünstigten noch verbleibenden Gegenstände an den Nacherben zu übertragen. Im Gegensatz zur nicht-befreiten Vorerbschaft wird der Vorerbe nicht mit der Auflage belastet, den zugewendeten Gegenstand für den Nacherben zu erhalten.

Außerdem können sich die Ehegatten durch eine vertragliche Erbeinsetzung gegenseitig schützen.

\subsection{Vertragliche Erbeinsetzung}

Verträge über einen noch nicht eröffneten Nachlass sind im französischen Recht grundsätzlich untersagt (prohibition des pactes sur succession future). Ausnahmsweise erlaubt der Code civil drei Formen von vertraglichen Erbeinsetzungen (institution contractuelle oder donation de biens à venir):

- Die vertragliche Erbeinsetzung durch Dritte zugunsten künftiger Ehegatten in deren Ehevertrag (Art. 1082 ff. des Code civil);

- Die vertragliche Erbeinsetzung zwischen künftigen Ehegatten im Ehevertrag (Art. 1091 ff. des Code civil);

- Die vertragliche Erbeinsetzung zwischen Ehegatten während der Ehe (Art. 1096 des Code civil).

In der Praxis tritt die letzte Form von vertraglicher Erbeinsetzung häufig auf. Sie wird als donation entre époux oder donation au dernier vivant bezeichnet. Sie erfordert eine notarielle Beurkundung. Sie ist frei widerruflich.

Die Testierfreiheit und somit die Möglichkeit, den Ehegatten $\mathrm{zu}$ bevorzugen, sind vom Pflichtteilsrecht beschränkt.

\section{Pflichtteilsrecht}

Das französische Noterbrecht (réserve héréditaire) gewährt einen echten Eigentumsanteil am Nachlass und ist damit Teil des Nachlasses.

Seit der Reform des 23. Juni 2006 enthält Art. 912 des Code civil eine gesetzliche Definition des Pflichtteils: „Der Pflichtteil ist ein Teil des Nachlassvermögens, den das Gesetz bestimmten Erben, genannt Noterben, lastenfrei zubilligt, wenn diese zur Erbfolge berufen sind und die Erbschaft annehmen."

Der Pflichtteil zählt zum französischen ordre public, nicht aber zum internationalen ordre public. ${ }^{9}$

\subsection{Pflichtteilsberechtigte Personen und Höhe des Pflichtteils}

Pflichtteilsberechtigt sind in erster Linie die Abkömmlinge des Erblassers. Bei Vorversterben oder Ausschlagung eines Kindes treten dessen Abkömmlinge im Wege des Eintrittsrechts (représentation) an seine Stelle.

9. Zwei Entscheidungen der Cour de cassation vom 27. September 2017 (Nr. 16-17.198 und 16-13.151). 
Art. 913 des Code civil bestimmt die frei verfügbare Quote (quotité disponible). Diese hängt von der Anzahl der Kinder ab:

- Ist ein Kind vorhanden, beträgt der frei verfügbare Teil die Hälfte (1/2) des Nachlasses;

- Bei zwei Kindern beträgt der frei verfügbare Teil ein Drittel (1/3) des Nachlasses;

- Bei drei und mehr Kindern beträgt der frei verfügbare Teil ein Viertel (1/4) des Nachlasses.

Die Höhe des Pflichtteils ergibt sich aus dem Umkehrschluss:

- $\quad$ Ein Kind: $1 / 2$ des Nachlasses;

- Zwei Kinder: 2/3 des Nachlasses;

- Drei Kinder und mehr: 3/4 des Nachlasses.

Unter Ehegatten gilt eine gesonderte Quote (quotité disponible spéciale entre époux). Gemäß Art. 1094-1 des Code civil kann der Erblasser zugunsten seines Ehegattens entweder über die nach Art. 913 bestimmte Quote oder über den Nießbrauch des gesamten Nachlasses oder über ein Viertel zu Eigentum und drei Viertel zu Nießbrauch verfügen.

Der überlebende Ehegatte ist nur wenn der Erblasser keine Abkömmlinge hinterlässt (Art. 914-1 des Code civil) pflichtteilsberechtigt. Sein Pflichtteil beträgt dann ein Viertel (1/4) des Nachlasses.

Mit der Reform des 23. Juni 2006 ist der Pflichtteil der Aszendenten weggefallen.

\subsection{Ermittlung des Pflichtteils}

Gemäß Art. 922 des Code civil wird für den vorhandenen Nachlass dessen Wert zum Zeitpunkt des Todes zugrunde gelegt. Nachlassschulden werden abgezogen. Miteinzurechnen sind die Gegenstände, die zu Lebzeiten durch Schenkungen veräußert wurden.

Die Überschreitung der frei verfügbaren Quote führt nicht zur Unwirksamkeit der Verfügung. Den Noterben steht eine Herabsetzungsklage (action en réduction) gegen exzessive Zuwendungen zu. Die Abfindung findet grundsätzlich in Form von Geldzahlungen statt.

Gemäß Art. 924-4 Abs. 4 des Code civil kann die Herabsetzungsklage auch gegen einen Dritterwerber geltend gemacht werden, wenn eine Immobilie von dem Begünstigten weiterveräußert wurde. Die Noterben können in der Schenkung oder im Kaufvertrag der Übertragung zustimmen und auf das Verfolgungsrecht gegenüber dem Dritterwerber verzichten.

Der Anspruch kann entweder einvernehmlich oder gerichtlich durchgesetzt werden. Seit dem 1. Januar 2007 beträgt die Verjährungsfrist der Herabsetzungsklage fünf Jahre ab Nachlasseröffnung oder zwei Jahre ab Kenntnis der Pflichtteilsverletzung, längstens jedoch zehn Jahre ab dem Erbfall (Art. 921 des Code civil).

\subsection{Pflichtteilsverzicht}

Den Pflichterben steht die Möglichkeit $\mathrm{zu}$, einer pflichtteilsverletzenden Verfügung nach dem Tod des Erblassers zuzustimmen (consentement à exécution). Eine solche Zustimmung bedarf keiner besonderen Formvorschrift.
Die Reform des 23. Juni 2006 hat mit den Art. 929 ff. des Code civil auch einen vorzeitigen - vor dem Tod des Erblassers erfolgenden - Verzicht auf die Herabsetzungsklage (renonciation anticipée à l'action en réduction) ermöglicht. Der Verzicht muss durch zwei Notare beurkundet werden. Dabei kann ein Notar von den Beteiligten frei gewählt werden, während der zweite Notar durch den Präsidenten der zuständigen Notarkammer bestimmt werden muss. Wegen dieses strengen Formalismus darf der vorzeitige Verzicht auf die Herabsetzungsklage nach herrschender Meinung nicht im Ausland unterzeichnet werden.

\section{Schlussbemerkungen}

Der überlebende Ehegatte hat im französischen Recht eine relativ starke Stellung: Er ist sowohl kraft des Güterstandes als auch kraft des Erbrechts am Vermögen des Erblassers beteiligt. Seine Stellung kann auch verbessert werden, wenn die Ehegatten entweder einen Ehevertrag mit güterrechtlichen Vorteilszuweisungen unterzeichnen oder sie Verfügungen von Todes wegen durch ein Testament oder eine vertragliche Erbeinsetzung treffen. Die Möglichkeit, den Ehegatten zu bevorzugen, wird aber vom Pflichtteilsrecht der Abkömmlinge beschränkt. 